\title{
CENDL project, the chinese evaluated nuclear data library
}

\author{
Zhigang Gea , Haicheng Wu, Guochang Chen, and Ruirui Xu \\ China Nuclear Data Center, China Committee of Nuclear Data, Key Laboratory of Nuclear Data Measurement and Evaluation, \\ China Institute of Atomic Energy, PO Box 275(41), Beijing 102413, China
}

\begin{abstract}
The status of Chinese Evaluated Nuclear Data Library (CENDL) and the relevant CENDL project are introduced in this paper. Recently, a new version CENDL-3.2b0 was being prepared on the basis of the previous CENDL-3.1. The data in the light and actinide nuclide regions are updated from CENDL-3.1, and the new evaluations and calculations are performed mainly around structure and fission product nuclide regions. Covariance was also evaluated for structure and actinide nuclides. At the same time, the methodologies are systematically developed to fulfil the requirements of evaluations for CENDL-3.2b0. The updated nuclear reaction models for light and middle-heavy nuclides, non-model dependent nuclear data evaluation, covariance evaluation approaches, systematics, and integral validation system of nuclear data are incorporated in present CENDL project. The future developments are also planned.
\end{abstract}

\section{Introduction}

With the current prosperity and development of nuclear applications in China, more requirements of the fundamental nuclear data are proposed from Chinese domestic users. In order to meet the diversified requirements and produce more high quality data to users, a CENDL project has been carrying out in China Nuclear Data Centre (CNDC), and China Nuclear Data Coordination Network (CNDCN) to systematically develop the methodologies in nuclear data evaluation and measurement. As the most important output of CENDL project, different versions of Chinese Evaluated Nuclear Data Library (CENDL) library (CENDL2, 2.1, 3.1) were released in the past, and used for some domestic and abroad users.

After several years effort, a new version of CENDL (CENDL-3.2b0) is nearly obtained, which involves the complete set of neutron induced reaction data below neutron energy at $20 \mathrm{MeV}$. activation, decay data and fission yield files etc. Although is not evolved into a very larger library comparing to previous vision CENDL-3.1 [1] in scale, but a lot of new data are included and previous defects are removed.

The paper is composed as follows. At first, the status of CENDL-3.2b0 is outlined in Sect. 2; the features of evaluations for each mass region are briefly discussed. The related methodologies are presented in Sect. 3; The all status are summarized in Sect. 4.

\section{Status of CENDL-3.2b0}

CENDL-3.2b0 is the fruit of most members in CNDC and CNDCN. Currently, 250 elements in total are contained in CENDL-3.2b0, and among them, about 60 elements are newly evaluated and updated from CENDL-3.1, and the evaluations of covariance for 12 elements are provided. The detailed elements are listed in Table 1.

a e-mail: gezg@ciae.ac.cn
The main process of revises and modifications will be presented in the following subsections.

\subsection{Light nuclei}

The main modifications in this mass region are for the neutron data of ${ }^{6,7} \mathrm{Li}$. A code FDRR based on the full diagonal R-matrix theory is used to perform the theoretical calculation. The neutron data for ${ }^{6} \mathrm{Li}$ was produced through this code and the fitted values of ${ }^{6} \mathrm{Li}(\mathrm{n}, \mathrm{t})$ cross sections are exampled in Fig. 1 to show good coincidence between the present calculation and experimental data available. In addition, the angular distributions of elastic scattering of $\mathrm{n}+{ }^{7} \mathrm{Li}$ system are updated with the new Legendre fittings to the experimental data. As a result, in Fig. 2, it can be observed the fitted values can meet with the experimental data exactly comparing to the other recommended data files.

\subsection{Structure and fission product nuclei}

In this region, ${ }^{23} \mathrm{Na},{ }^{27} \mathrm{Al},{ }^{32,33,34,36} \mathrm{~S},{ }^{40} \mathrm{Ca},{ }^{56} \mathrm{Fe},{ }^{181} \mathrm{Ta}$ and $\mathrm{W}$ stable isotopes are re-calculated by the UNF code [2]. The non-model dependent evaluations are performed thoroughly to the cross sections with abundant experimental data. Figure 3 shows the experimental data evaluation result for ${ }^{32} \mathrm{~S}(\mathrm{n}, \mathrm{p})$ and the relevant modification in theoretical calculation. It can be observed that the present recommended nuclear data follows the evaluated cross sections rigorously.

The evaluation of all measured data for the very important structure material ${ }^{56} \mathrm{Fe}$, which was included in the CIELO [3] project, was performed in the fast neutron energy range. Especially for the inelastic scattering cross section, multiple ways were applied to clarify the discrepancy in the energy range between 9-11 MeV (Fig. 4). Gamma-ray production cross sections provide a way to gain experimental information about the inelastic cross section. Also the nonelastic, $(n, 2 n)$ and

(C) The Authors, published by EDP Sciences. This is an Open Access article distributed under the terms of the Creative Commons Attribution License 4.0 (http://creativecommons.org/licenses/by/4.0/). 
Table 1. The nuclei of CENDL-3.2b0.

\begin{tabular}{|c|c|}
\hline & Content of Nuclide \\
\hline $\begin{array}{l}\text { Light } \\
\text { Elements }\end{array}$ & ${ }^{1-3} \mathrm{H},{ }^{3,4} \mathrm{He},{ }^{6,7} \mathrm{Li},{ }^{9} \mathrm{Be},{ }^{10,11} \mathrm{~B},{ }^{12} \mathrm{C},{ }^{14} \mathrm{~N},{ }^{16} \mathrm{O},{ }^{19} \mathrm{~F}$ \\
\hline $\begin{array}{l}\text { Structure } \\
\text { Materials }\end{array}$ & $\begin{array}{l}{ }^{23} \mathrm{Na},{ }^{24-26} \mathrm{Mg}^{27}{ }^{27} \mathrm{Al},{ }^{28-30} \mathrm{Si}^{31}{ }^{31},{ }^{32,33,34,36} \mathrm{~S},{ }^{0} \mathrm{Cl},{ }^{0} \mathrm{~K}, \\
{ }^{40} \mathrm{Ca},{ }^{46-50} \mathrm{Ti},{ }^{0} \mathrm{~V},{ }^{50,52-54} \mathrm{Cr},{ }^{55} \mathrm{Mn},{ }^{54,56-58} \mathrm{Fe},{ }^{59} \mathrm{Co}, \\
{ }^{58,60-62,64} \mathrm{Ni}^{0,63,65} \mathrm{Cu},{ }^{0} \mathrm{Zn},{ }^{0} \mathrm{Ge},{ }^{90-92,94,96} \mathrm{Zr},{ }^{92,94-} \\
{ }^{98,100} \mathrm{Mo},{ }^{0,107,109} \mathrm{Ag},{ }^{0} \mathrm{Cd},{ }^{0} \mathrm{Sn},{ }^{174,176-180} \mathrm{Hf},{ }^{181} \mathrm{Ta}, \\
{ }^{180,182,183,184,186} \mathrm{~W},{ }^{197} \mathrm{Au},{ }^{0} \mathrm{Hg},{ }^{0} \mathrm{Tl},{ }^{204,206-208} \mathrm{~Pb},{ }^{209} \mathrm{Bi}\end{array}$ \\
\hline $\begin{array}{l}\text { Fission } \\
\text { Products } \\
\& \\
\text { Medium } \\
\text { Elements }\end{array}$ & $\begin{array}{l}{ }^{69,71} \mathrm{Ga},{ }^{70-78} \mathrm{Ge},{ }^{75,77,79} \mathrm{As},{ }^{83,84,85,86,87} \mathrm{Kr},{ }^{85,87} \mathrm{Rb},{ }^{88-} \\
{ }^{90} \mathrm{Sr},{ }^{89,91} \mathrm{Y},{ }^{93,95} \mathrm{Zr},{ }^{93,95} \mathrm{Nb},{ }^{99} \mathrm{Tc},{ }^{99-105} \mathrm{Ru}, \\
{ }^{103,105} \mathrm{Rh},{ }^{105,108} \mathrm{Pd},{ }^{113} \mathrm{Cd},{ }^{113,115} \mathrm{In},{ }^{112,114-120,122,124} \mathrm{Sn}, \\
{ }^{121,123,125} \mathrm{Sb},{ }^{130} \mathrm{Te},{ }^{127,129,135},{ }^{123,124,129,131,132,133,134-} \\
{ }^{136} \mathrm{Xe},{ }^{133-135,137} \mathrm{Cs},{ }^{130,132,134-138} \mathrm{Ba},{ }^{139} \mathrm{La},{ }^{136,138,140-} \\
{ }^{142,144} \mathrm{Ce}^{141}{ }^{141} \mathrm{Pr},{ }^{142-148,150} \mathrm{Nd},{ }^{147,148,148 m, 149} \mathrm{Pm},{ }^{144,147-} \\
{ }^{152,154} \mathrm{Sm},{ }^{151,153-155} \mathrm{Eu},{ }^{152,153,154-158,160} \mathrm{Gd},{ }^{164} \mathrm{Dy}\end{array}$ \\
\hline Actinides & $\begin{array}{l}{ }^{232} \mathrm{Th},{ }^{232-240,241} \mathrm{U},{ }^{236-239} \mathrm{~Np},{ }^{236-246} \mathrm{Pu},{ }^{240-244,242 \mathrm{~m}} \mathrm{Am}, \\
{ }^{249} \mathrm{Bk},{ }^{249} \mathrm{Cf}\end{array}$ \\
\hline
\end{tabular}

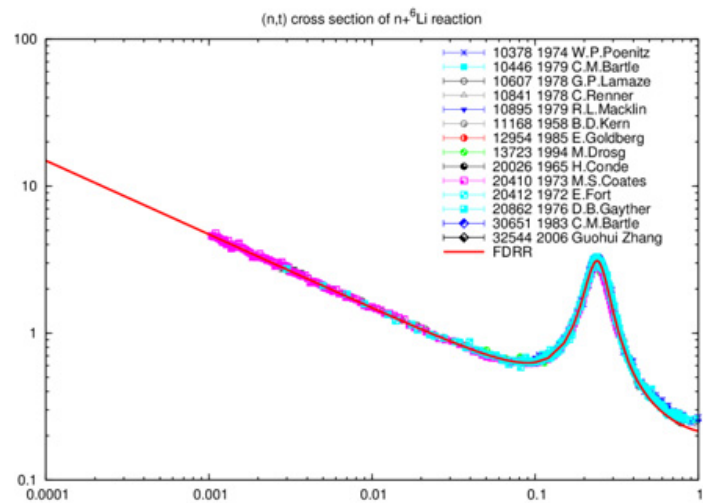

Figure 1. The comparisons of calculated ${ }^{6} \mathrm{Li}(\mathrm{n}, \mathrm{t})$ cross section with FDRR and the experimental data available.

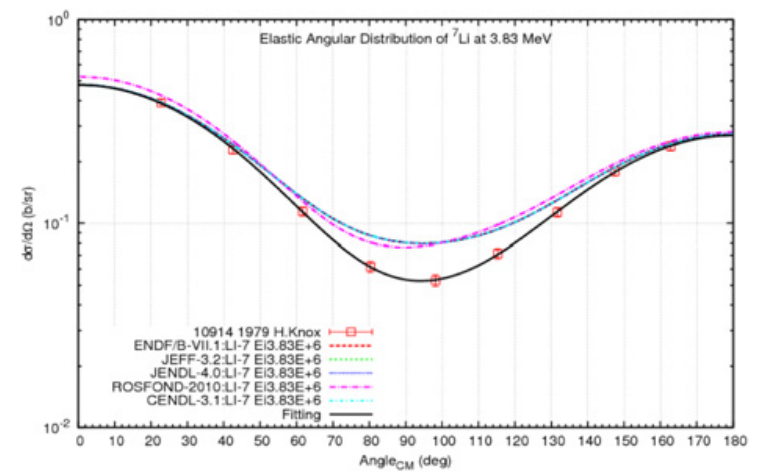

Figure 2. The comparisons of the fitted angular distributions of elastic scattering, experimental data and other recommendations.

(n, a) cross sections were improved in the mode calculation due to correction for the missing inelastic scatting cross section to the first excited level, and new experimental data respectively.

\subsection{Actinides}

The actinides ${ }^{241} \mathrm{Am},{ }^{234,235} \mathrm{U},{ }^{237} \mathrm{~Np},{ }^{233} \mathrm{Th} \quad{ }^{232} \mathrm{Th}$, ${ }^{232-240,241} \mathrm{U},{ }^{236-239} \mathrm{~Np},{ }^{236-246} \mathrm{Pu},{ }^{240-244,242 \mathrm{~m}} \mathrm{Am},{ }^{249} \mathrm{Bk}$, ${ }^{249} \mathrm{Cf}$ have been updated with the calculations of FUNF [4] code and new experimental information. Figures 5-6 show

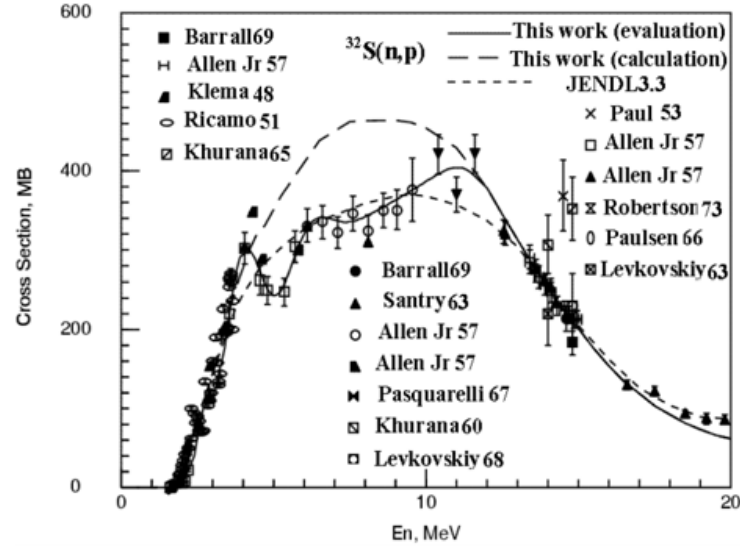

Figure 3. The comparisons of calculated (dashed line) and evaluated (solid line) ${ }^{32} \mathrm{~S}$ (n, p) cross section and the experimental data available.

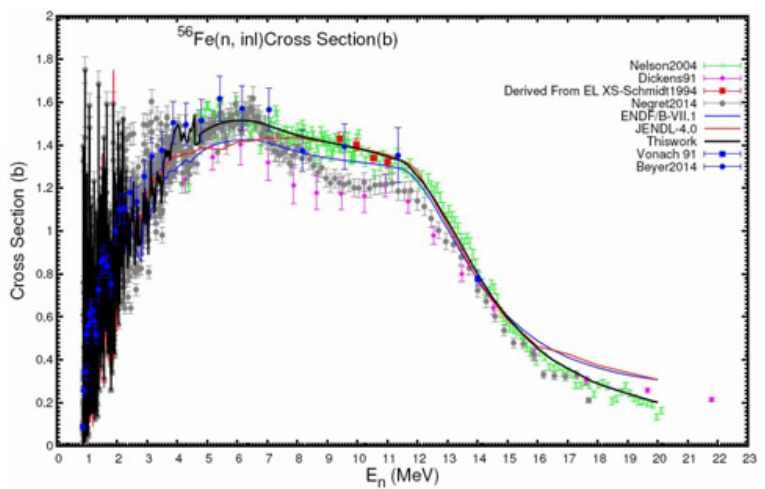

Figure 4. The updated evaluation (black line) of ${ }^{56} \mathrm{Fe}(\mathrm{n}, \mathrm{inl})$ cross section compared with exp. and other libraries.

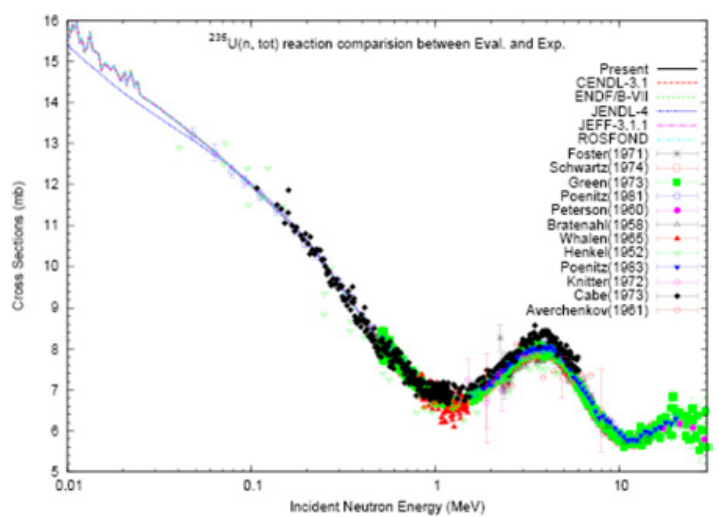

Figure 5. Comparison of re-evaluated (n,tot) CS with measured data and other evaluations for ${ }^{235} \mathrm{U}$.

the new evaluation compeared with other evaluations and experimental data.

\subsection{Benchmark test}

The C32b0 has been tested with all the criticality benchmarks in a nuclear data validation system ENDITS-1.0. Some of the comparison results are shown here.

The validation results shown that the data for ${ }^{233,235} \mathrm{U}$, ${ }^{232} \mathrm{Th}$ have been improved significantly. For ${ }^{235} \mathrm{U}$, by loading the fission cross sections from IAEA 2006 standard [5], reevaluating the $\alpha$ values and revising the resolved resonance parameters according to the nuclear 


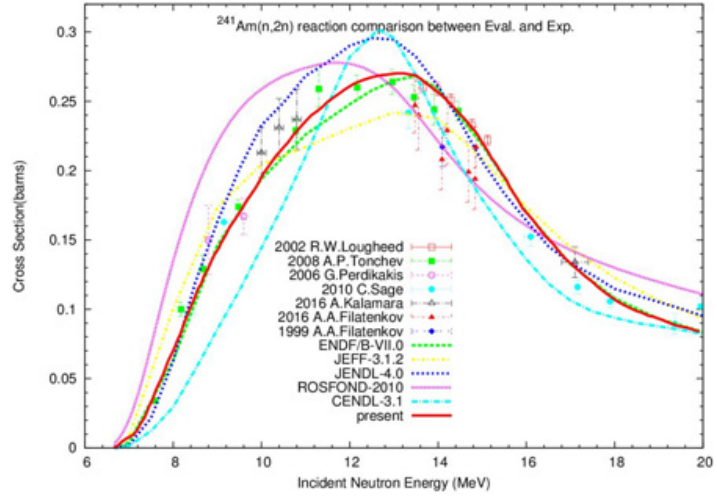

Figure 6. The comparison of evaluated ${ }^{241} \mathrm{Am}(\mathrm{n}, 2 \mathrm{n})$ with other data.

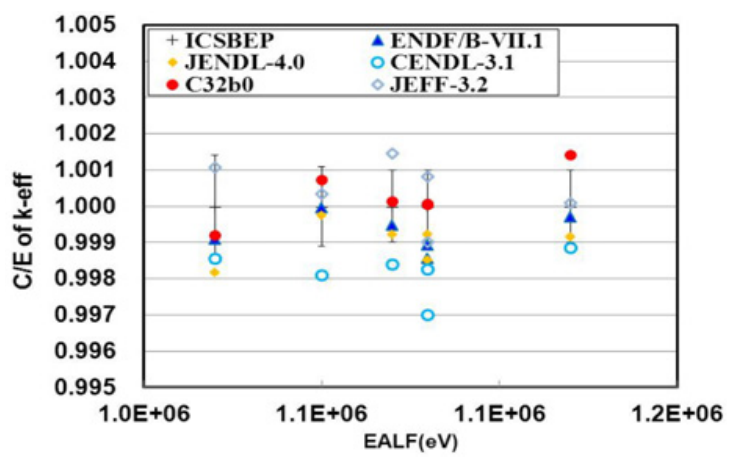

Figure 7. The comparison of the $\mathrm{C} / \mathrm{E}$ values of the $\mathrm{k}_{\text {eff }}$ for the bare and uranium reflected ${ }^{233} \mathrm{U}$ spheres.

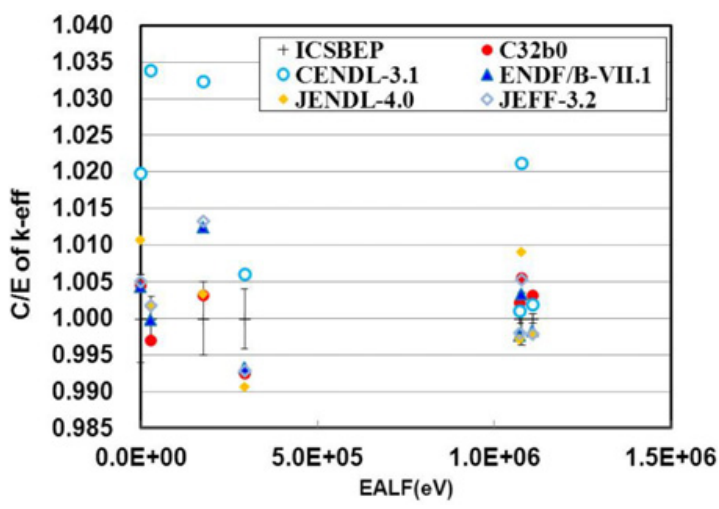

Figure 8. The $\mathrm{C} / \mathrm{E}$ results of fast and intermediate spectra benchmarks sensitive to ${ }^{232} \mathrm{Th}$ (KBR and Thor).

data adjustment based on the selected HMT benchmarks, the predictions of the $k_{\text {eff }}$ values for the uranium fuelded system have been significantly improved. As the results shown in Table 1 , the normalized $\chi^{2}$ values for most of the systems calculated with the $\mathrm{C} 32 \mathrm{~b} 0$ have been generally improved compared to CENDL-3.1. For all the uranium fueled benchmarks in the ENDITS-1.0, C32b0 gives the best prediction of $\mathrm{k}_{\mathrm{eff}}$ values compared with the other libraries. For the bare and uranium reflected ${ }^{233} \mathrm{U}$ spheres, as shown in Fig. 7, the $\mathrm{C} / \mathrm{E}$ values closed to unit due to the revised ${ }^{233} \mathrm{U}(\mathrm{n}, \mathrm{inl})$ cross setions [6]. For the fast and intermediate spectra benchmark KBR and Thor, the $\mathrm{k}_{\mathrm{eff}}$ values are sensitive to the data of ${ }^{232} \mathrm{Th}$, the $\mathrm{C} / \mathrm{E}$ values of the $\mathrm{k}_{\mathrm{eff}}$ have been improved significantly by revised ${ }^{232} \mathrm{Th}(\mathrm{n}, \gamma)$ cross sections.
Table 2. Comparison of $\left(\chi^{2}-1\right)$ of $k_{\text {eff }}$ values for uranium fueled criticality benchmarks.

\begin{tabular}{|l|l|c|l|c|c|l|}
\hline Type & Cores & C32b0 & C31 & B71 & J40 & F32 \\
\hline HCF & 5 & 1.2 & 0.8 & 2.6 & 2.0 & 1.8 \\
\hline HCI & 8 & 825 & 843 & 915 & 642 & 741 \\
\hline HCM & 18 & $\mathbf{1 . 1}$ & 47.4 & 136 & 25.2 & 86.9 \\
\hline HCT & 43 & $\mathbf{2 . 9}$ & 4.3 & 2.5 & 8.1 & 2.5 \\
\hline HMF & 151 & $\mathbf{4 6 0}$ & 539 & 500 & 526 & 560 \\
\hline HMI & 6 & 48.6 & 40.3 & 24.9 & 20.4 & 95.4 \\
\hline HMT & 89 & $\mathbf{2 9 . 4}$ & 130 & 47.0 & 48.8 & 23.3 \\
\hline HST & 118 & 2.1 & 1.9 & 1.7 & 1.6 & 1.9 \\
\hline ICF & 2 & $-\mathbf{1 . 0}$ & 39.6 & 4.9 & 128 & 10.3 \\
\hline ICI & 2 & $-\mathbf{1 . 0}$ & 132 & -0.5 & 2.6 & 0.2 \\
\hline ICT & 33 & $-\mathbf{0 . 2}$ & 1.9 & 0.6 & 4.6 & 0.7 \\
\hline IMF & 17 & $\mathbf{1 . 6}$ & 10.4 & 2.6 & 61.3 & 9.2 \\
\hline LCT & 122 & 6.3 & 4.2 & 3.5 & 3.4 & 3.1 \\
\hline LMT & 26 & -0.4 & 0.0 & -0.3 & -0.1 & 0.4 \\
\hline LST & 58 & $\mathbf{2 . 0}$ & 6.2 & 3.7 & 2.6 & 2.4 \\
\hline U235 & 698 & $\mathbf{1 1 4}$ & 145 & 128 & 130 & 135 \\
\hline
\end{tabular}

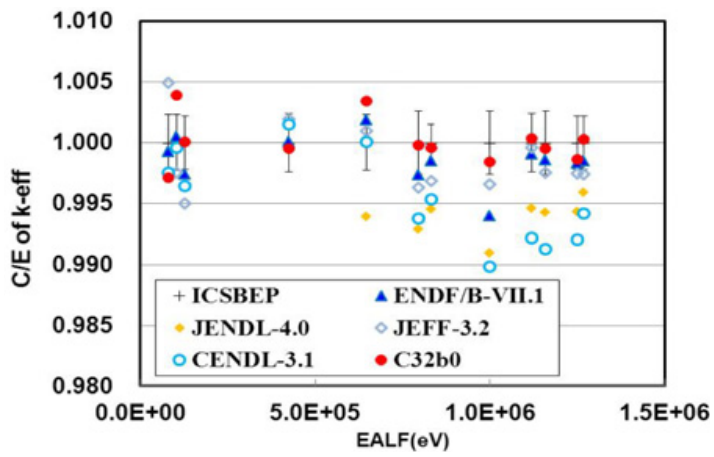

Figure 9. The comparison of the $\mathrm{C} / \mathrm{E}$ values of the benchmarks that are sensitive to ${ }^{56} \mathrm{Fe}$ (HMF13, 21, ICF1, IMF5, 10, PMF25, $26,28,32)$.

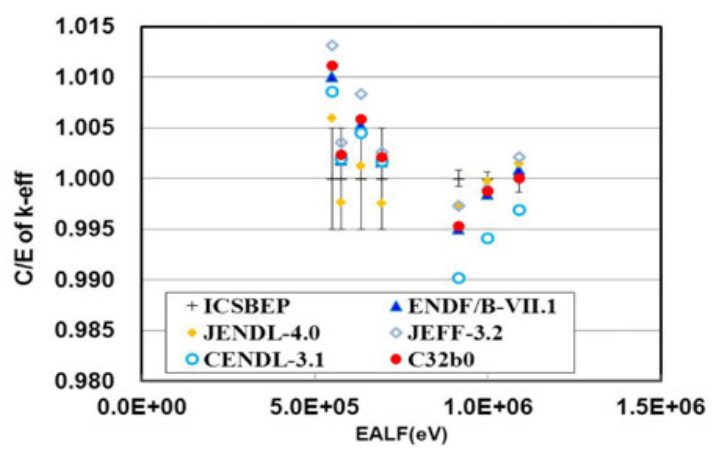

Figure 10. The comparison of the benchmark results for the MET-FAST benchmarks that sensitive to tungsten (HMF3, PMF5, UMF4).

The performance of the structure material of $\mathrm{C} 32 \mathrm{~b} 0$ has also been improved. As shown in Fig. 9, the serious underprediction of the $\mathrm{k}_{\mathrm{eff}}$ values by CENDL-3.1 for the fast spectrum benchmarks that are sensitive to the data of ${ }^{56} \mathrm{Fe}$ have been eliminated and get closed to unit by using the new data of ${ }^{56} \mathrm{Fe}$. In Fig. 10, the under predictions of the $\mathrm{C} / \mathrm{E}$ values of $\mathrm{k}_{\mathrm{eff}}$ for the tungsten reflected fast criticality benchmarks have also been alleviated.

Generally, the benchmark results show that the predictions of the $\mathrm{k}_{\text {eff }}$ with the new revision have been improved significantly compared to the CENDL-3.1. 

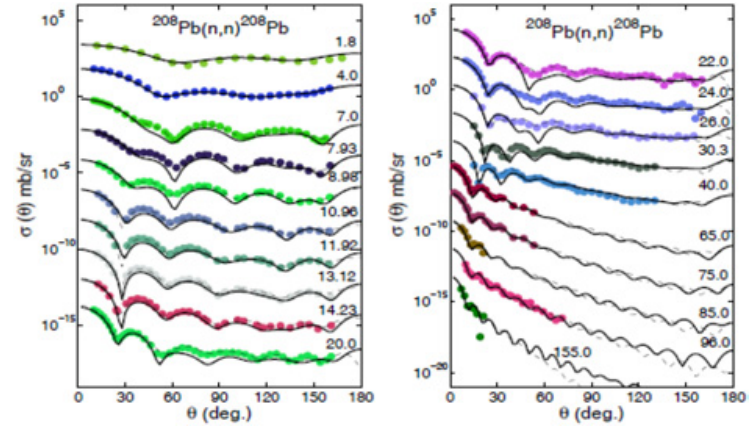

Figure 11. The comparisons of predicted angular distributions of elastic scattering (solid line) with results of KD potentials (dashed line) and the experimental data (points) for $\mathrm{n}+{ }^{208} \mathrm{~Pb}$ at neutron incident energies from $1.8 \mathrm{MeV}$ to $155 \mathrm{MeV}$.

\section{Methodologies in CENDL}

Many methodologies are involved in the CENDL project to improve the abilities of nuclear data evaluation in China. Overall, the nuclear data evaluations based on nuclear reaction and structure theory, non-model nuclear data evaluation approach, the covariance evaluation approach, and the integral validation System are all systematically developed in the past several years. They will be introduced in this section.

\subsection{Nuclear reaction model and codes}

The UNF series codes for light, middle-heavy and actinide nuclei have been developed for many years in CNDC. Most of the popular reaction models are compiled in UNF, the optical model potentials, unified H-F and exiton model, pick up mechanism, Kalbach systematics, complex particle emission, recoil nuclide and so on. In order to extend its ability in the unstable nuclei predictions, many ingredients including optical model potentials, level densities, giant dipole resonance et al., were further investigated in a more rigorous way, moreover, the parameters based on a microscopic fundamental theory will increase the confidence of users to apply the predictions. For example, many new microscopic optical potential (MOP) were generated and the widely-used optical models were incorporated in the present UNF to offer users with more options so as to assess better predictions. Figure 11 shows the predictions for the angular distributions of neutron elastic scattering from ${ }^{208} \mathrm{~Pb}$ by using the latest derived nucleon-nucleus microscopic optical potential, which was based on the isospin dependent Dirac Brueckner H-F calculations and collaboratively developed by CNDC and Tuebingen University [7]. This potential can well reproduce the scattering observables, including the total neutron and proton reaction cross sections, angular distributions, analysing powers and spin rotation functions for mass region of $\mathrm{A}=12-208$ below $200 \mathrm{MeV}$. Moreover, considering the few freedom, this potential can provide an advanced framework for the description of scattering data for unstable nuclei and for studying nuclear structure models of exotic nuclei.

For neutron induced $1 \mathrm{p}$ shell nuclei reactions, the full and diagonal reduced R-matrix was utilized, and a code FDRR was being developed, and the neutron data for ${ }^{6} \mathrm{Li}$ was evaluated by FDRR, and satisfying results were obtained. Currently, besides the full diagonal reduced

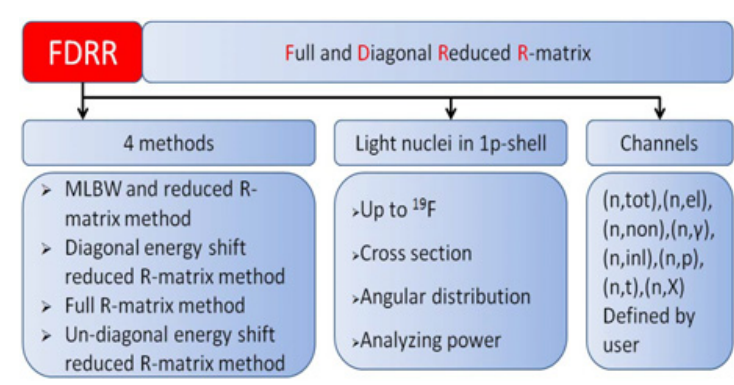

Figure 12. The scheme of FDRR code.

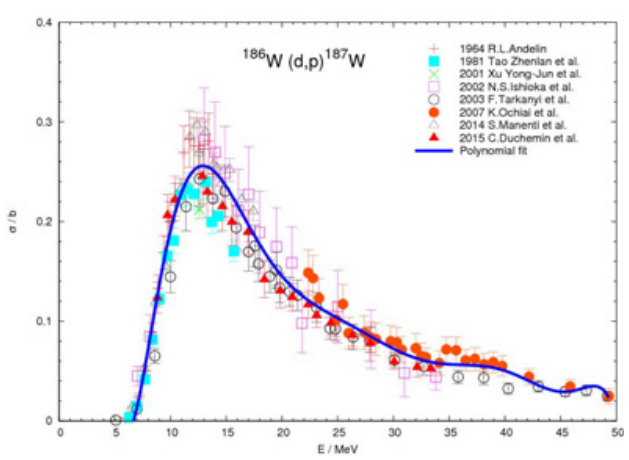

Figure 13. The excitation function evaluation for ${ }^{186} \mathrm{~W}(\mathrm{~d}, \mathrm{p})^{187} \mathrm{~W}$.

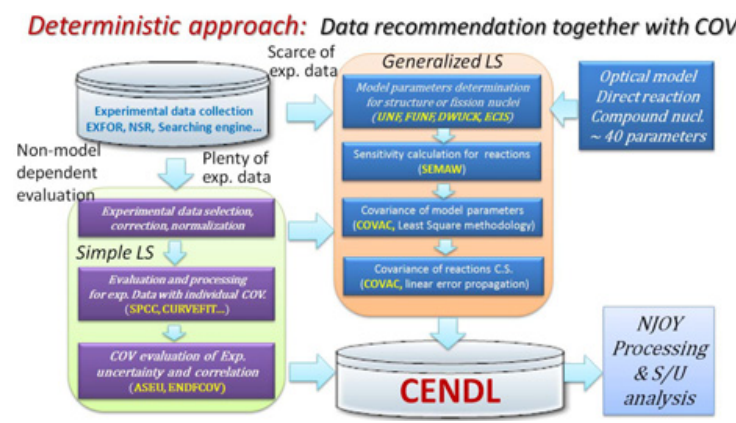

Figure 14. The scheme of covariance evaluation system of CENDL project.

R-matrix approach, other three methods are also employed in FDRR as shown in Fig. 12, various data and reactions can be considered as the same time.

\subsection{Non-model dependent evaluation}

The non-model dependent evaluation is the chief process in the evaluation to the quantities with abundant of experimental data. The final recommendation by evaluator should seriously consider all of the experimental data, especially to the important reactions et al. For example, the evaluation for ${ }^{186} \mathrm{~W}(\mathrm{~d}, \mathrm{p}){ }^{187} \mathrm{~W}$ was performed through the experimental data correction, normalization, non-model dependent polynomial fitting et al.. The obtained results in Fig. 13 keep in very good agreement with the experimental data in the whole energy region.

Some attempts have also being performed recently to improve CNDC evaluation system, such as the simultaneous evaluation, systematics, et al.

\subsection{Nuclear data covariance evaluation}

Nuclear data covariance remains a big issue to consider the uncertain elements in the nuclear data evaluation. 


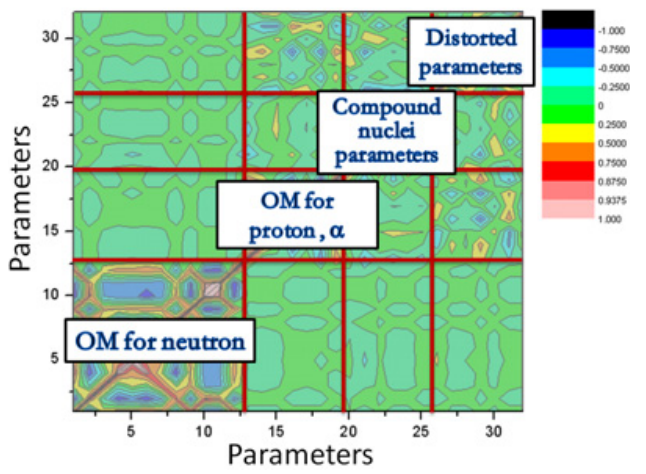

Figure 15. The obtained covariance of model parameters of $\mathrm{n}+{ }^{48} \mathrm{Ti}$ calculation in the model-dependent case.

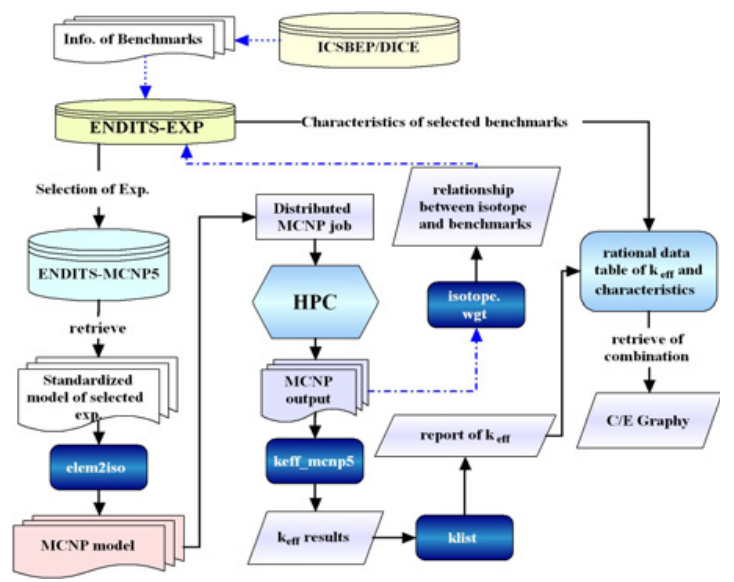

Figure 16. The MCNP working flowchart of ENDITS-1.0.

In general, the uncertainties in data evaluation are located in the data measurement, evaluation, model calculation and others. Therefore, the chief purpose of covariance evaluation is to 'honestly' propagate any related uncertainty in nuclear data recommendation process to covariance in a scientific way, but not only the purpose to including REAL values within the boundary.

The systematic evaluation scheme of covariance has been built based on the deterministic Least Square (LS) approach, which have already been taken to serve in the covariance evaluation for CENDL-3.2b0.

Figure 15 shows the covariance of model parameters for $\mathrm{n}+{ }^{48} \mathrm{Ti}$ calculations, the data in different regions denote the relevant correlations between parameters. The covariance for parameters exhibits the correlations among various models parameters. It should also be mentioned that the results in Fig. 9 incorporate the uncertain sources from experimental data and model both.

The covariance scheme can be utilized to the middleheavy, actinide nuclei within the fast neutron energy region. The cross sections of (n,tot), $(n, e l),(n, \gamma),(n, i n l)$, $(n, p),(n, 2 n),(n, n p),(n, n \alpha),(n, 3 n),(n, f)$ et al., are involved in the consideration.

\subsection{Integral validation system}

A nuclear data validation system ENDITS-1.0 has been developed. The system is composed by following component:

- ENDITS-EXP, a rational database develop based on the ICSBEP2006 [8], which contains the experiment

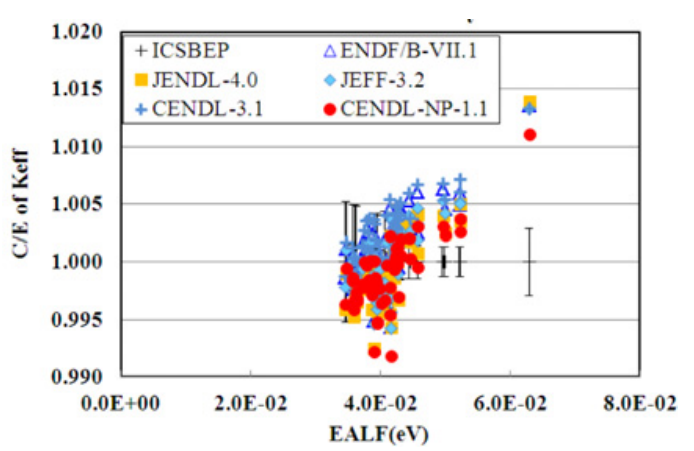

(a) All the selected LEU-SOL-THERM benchmarks

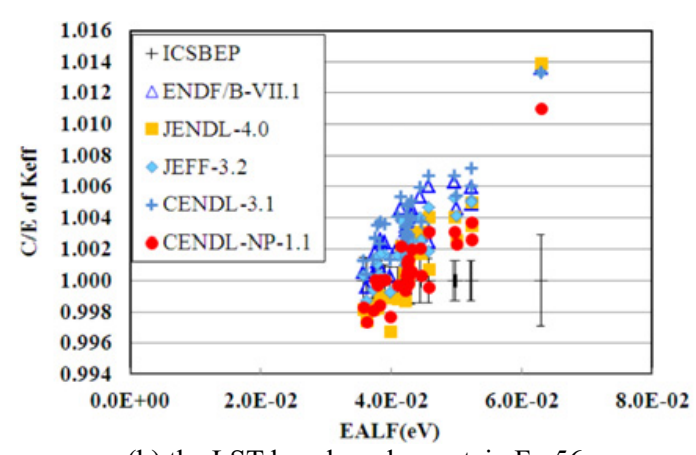

(b) the LST benchmarks contain Fe-56

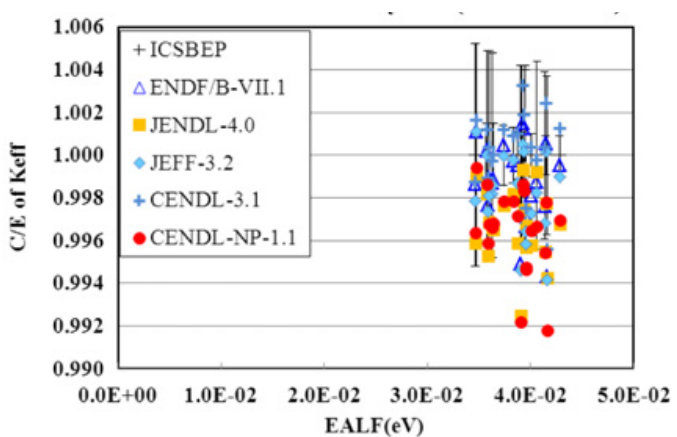

(c) the LST benchmarks do not contain Fe-56

Figure 17. $\mathrm{C} / \mathrm{E}$ trend of $\mathrm{k}_{\mathrm{eff}}$ against EALF for LEU-SOLTHERM benchmarks.

information of benchmarks and isotope importance in criticality calculation for each selected experiment.

- ENDITS-MCNP: a set of standardized MCNP models, from where MCNP input decks can be generated in batch for different nuclear data library.

- Auxiliary codes, including the function of generating MCNP input decks in batch from standardized model, collecting and reporting $\mathrm{k}_{\mathrm{eff}}$ results in batch, and construct relationship between isotope and benchmarks.

The ENDITS-1.0 system contains characteristics of 1261 criticality benchmarks. C/E trend of $\mathrm{k}_{\mathrm{eff}}$ again spectra index EALF filtered by experiment characteristics such as fuel type, spectrum type, reflector materials, isotope important/sensitivity et al. Which can helps testers to identify the evaluation data need to improve conveniently. Figure 17 shows the trend analysis with a relational database. A C/E trend of $\mathrm{k}_{\text {eff }}$ against spectral index EALF for the LST system was observed in all evaluated library CENDL-3.1, CENDL-NP-1.1, ENDF/B-VII.1 [9], JENDL-4.0 [10] and JEFF-3.2 [11]. By include and exclude the results from the 
LST benchmarks contain ${ }^{56} \mathrm{Fe}$, the trend can be identified as ${ }^{56} \mathrm{Fe}$ correlated preliminary. Then, sensitivity analysis and data adjustment with the typical benchmarks selected from a trend can be taken as next step to further analysis and locate to more detailed reactions or energy regions.

Currently, only criticality and shielding benchmarks are included in the ENDITS-1.0. Other experiments, such as effective delayed neutron experiment, will be included in future.

\section{Summary}

The latest CENDL-3.2b0 and the recent research work in CENDL project are introduced in this paper. Besides the achievement above, some aspects are now designed in our future plan. First of all, the current theoretical model code for nuclear reaction will be extended to unstable nuclei with the new ingredients of microscopic models, systematics and so on; secondly, more attention will be paid to discuss the key issues in current fission theory study; thirdly, the accuracy of nuclear data and covariance in CENDL library will be further improved, in particular to the actinides, which would be the main modification in the next CENDL version after CENDL-3.2b0. Simultaneously, the powerful approaches such as simultaneous evaluation will be utilized. The evaluation around RRR and URR region will be further developed in the future with the foundation of advanced neutron beam source in China. In addition, the photonuclear library and relevant methodology will be incorporated in the future.

The authors would like to thank the colleagues of CNDC and all members of CNDCN to make their great efforts to the nuclear data libraries establishment and the related methodology study. Moreover, the authors would like to show our acknowledgements to IAEA, NEA-DB and other nuclear data centres for their useful advices and kindly help and discussion.

\section{References}

[1] Z.G. Ge, Y.X. Zhuang, T.J. Liu, J.S. Zhang, H.C. Wu, Z.X. Zhao, H.H. Xia, Journal of the Korean Physical Society 59(2), 1052-1056 (2011)

[2] J.S. Zhang, Nucl. Sci. Eng. 142, 207 (2002)

[3] M.B. Chadwick et al., Nucl. Data Sheets 118, 11 (2014)

[4] Zhang Jingshang, User Manual of FUNF code. Atomic Energy Press 2005, ISBN 7-5022-3584-1

[5] STI-PUB-1291, International Atomic Energy Agency, vienna (2007)

[6] H. Wu, H. Zhang, Atomic Energy Science and Technology 46(10), 1158-1164 (2012)

[7] R.R. Xu, et al., Physical Review C 94, 034606 (2016); R.R. Xu, et al., Physical Review C 85, 034613 (2012)

[8] NEA Nuclear Science. Committee. NEA/NSC/DOC (95)04/I, Nuclear Energy Agency, OECD, Paris, France (2006)

[9] M.B. Chadwick, M. Herman, P. Oblozinsky, et al., Nuclear Data Sheets 112(12), 2887-2996 (2011)

[10] K. Shibata, O. Iwamoto, T. Nakagawa, et al., J. Nucl. Sci. Technol. 48(1), 1-30 (2011)

[11] JEFF and EFF/EAF working groups, JEFF-3.2 evaluated data library, http://www.oecd-nea.org/ dbforms/data/eva/evatapes/jeff_ 32 (2014) 